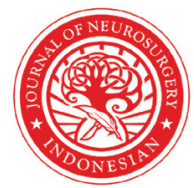

Indonesian Journal of Neurosurgery

\title{
Effect of pre-surgical factors against degenerative lumbal patient surgical actions: evaluation using modified oswestry disability index and rolland morris
}

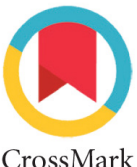

\author{
Yudha Fitrian Prasetyo ${ }^{1 *}$, Abdul Hafid Bajamal' ${ }^{1}$, Hari Basuki ${ }^{1}$
}

'Departement of Neurosurgery, Faculty of Medicine Airlangga University/ Dr. Soetomo General Academic Hospital, Indonesia

\section{*Corresponding to:}

Yudha Fitrian Prasetyo; Departement of Neurosurgery, Faculty of Medicine Airlangga University/ Dr. Soetomo General Academic Hospital, Indonesia; yudha.fp@gmail.com

Received: 2020-07-08

Accepted: 2021-03-13

Published: 2021-04-23

\section{ABSTRACT}

Background: Lower back pain is the most common complaint in humans in their lives with a variety of heterogeneous substances that vary in their epidemiological studies. Several disability scales have been developed and developed for the assessment of low back pain patients as a measure of outcome of therapy. One of the pre- and post-operative evaluation methods that are widely used is the Modified Oswestry Disability Index (MODI) and the Rolland Morris Disability Index. This disability scale is a disability scale that assesses daily personal and social functions using a questionnaire method and has been extensively reviewed by various study centers so that it is widely used as a guide for operative evaluation Objective: the study is aimed to knowing the effect of pre-surgical factors on surgical outcome in degenerative lumbar patients evaluated using the Modified Oswestry and Rolland Morris disability scales.

Methods: The study was conducted at the Dr. Soetomo General Academic Hospital by taking data from lumbar abnormality patients who were operated on in the period of 1 June 2018 until 30 June 2019. The data is processed by calculating the Modified Oswestry Disability Index score and the Rolland Morris Disability Index during pre and post-operative, then the variables are calculated with each categorical regression to assess the effect of each variable

Results: Total sample of 42 with 31 samples fulfilling the inclusion criteria. There are 19 male sex samples and 12 female sex samples. From the age variable data, obtained the most age range at the age of $40-60$ years. Whereas in the variable body mass index was obtained the most in samples with a range of values of $18.5-25$. The adjusted $R^{2}$ (coefficient of determination) result is 0.746 for MODI and adjusted $R^{2}$ (coefficient of determination) result of 0.513 for Rolland Morris Disability Scale.

Conclusion: There is an effect of sex, age, sitting working position and sacral slope factors on the outcome of surgical procedures in the degenerative lumbar patient evaluated with the Modified Oswestry disability scale. Whereas the age and sitting work position have an influence in surgical outcome in the patients evaluated with Rolland Morris scale. Number of intervention levels tend to have a significant effect on disability score difference.

Keywords: Lumbar Degenerative Disease, Disability Index, Modifies Oswestry, Rolland Morris

Cite This Article: Prasetyo, Y.F., Bajamal, A.H., Basuki, H. 2021. Effect of pre-surgical factors against degenerative lumbal patient surgical actions: evaluation using modified oswestry disability index and rolland morris. Indonesian Journal of Neurosurgery 4(1): 17-24. DOI: 10.15562/ijn.v4i1.140

\section{INTRODUCTION}

Lower back pain is the most common complaint in humans in their lives with a variety of heterogeneous substances that vary in their epidemiological studies. Temporality and topographic definitions are often not given clear boundaries. However, a study conducted by Dionne et al. Provides a limitation with the definition of pain felt in the lower part of rib 12 to the bottom of the gluteal sulcus which can limit activity for more than one day. ${ }^{1}$
Many environmental, personal, and anatomical factors influence the onset and course of lower back pain. With symptoms that can have a significant impact on the quality of life of patients, modifiable risk factors are very important to know and prevent disease, rehabilitation, and recurrence. $^{2}$

Lumbar degeneration dominates and is a major factor that triggers back pain. Practically, disc degeneration is related to the structural and biochemical changes of the disc caused mainly by increasing age and excessive physical burden. In recent years, hormonal, systemic, and even genetic factors have become predisposing factors that influence the course of spinal disease. $^{3}$

Risk factors and predisposing factors in patients with low back pain have been studied as pre-surgical factors for evaluation and prognosis. Factors that age, weight, occupation, activity, and even the educational status of the patient 
is associated as predictors of therapeutic outcomes. Various epidemiological studies from each region can be a useful comparative data for education in populations of patients with low back pain. $^{2}$

Operative therapy in cases of back pain produces varied but significant operating outcomes in improving the quality of life of patients. Preoperative factors are thought to play a large role in the prognosis of the disease both with operative and nonoperative therapy. Evaluation of results of operations in various study centers is documented with a variety of evaluation methods. ${ }^{4}$

Pain and disability are the main symptoms in lumbar degenerative diseases and are the reason for the majority of patients to undergo operative therapy. Methods of evaluating pain and disability before and after patients undergo operative action are needed. This evaluation method is often only based on patient complaints and is not well documented, especially at the study center. This scoring and evaluation method makes it a must at the center of the study to determine the regression of the pain scale and reduce the disability scale with clearer guidelines. ${ }^{5}$

Several disability scales have been developed and developed for the assessment of low back pain patients as a measure of outcome of therapy. One of the pre- and post-operative evaluation methods that are widely used is the Modified Oswestry Disability Index (MODI) and the Rolland Morris Disability Index. This disability scale is a disability scale that assesses daily personal and social functions using a questionnaire method and has been extensively reviewed by various study centers so that it is widely used as a guide for operative evaluation. ${ }^{6}$

\section{METHODS}

This research is an analytic observational study. The study was conducted at the Dr. Soetomo General Academic Hospital by taking data from lumbar abnormality patients who were operated on in the period of 1 June 2018 until 30 June 2019. The inclusion criteria for this study were degenerative lumbar patients who were admitted and underwent surgery at Dr. Soetomo General Academic Hospital.
Whereas the exclusion criteria were trauma patients or lumbar infections, patients who had undergone previous surgery, and patients or families of patients who were not willing to participate in the study.

This study uses primary data collected through data recording and through filling out questionnaires in the Dr. Soetomo General Academic Hospital ward. The data is processed by calculating the Modified Oswestry Disability Index score and the Rolland Morris Disability Index during pre and post-operative period, then the variables are calculated with each categorical regression to assess the effect of each variable

\section{RESULTS}

The study was conducted in June 2018 until June 2019 with a total sample of 42 with 31 samples fulfilling the inclusion criteria. All samples were willing to be the subject of research by answering the Modified Oswestry disability scale questionnaire and the Rolland Morris disability scale before surgery. Of the 31 samples obtained only 23 samples that have lumbosacral xray of AP-lateral view for measurement of pelvic parameters (sacral slope, pelvic tilt, pelvic incidence).

From anthropometric factors, there are 19 male sex samples and 12 female sex samples. From the age variable data, obtained the most age range at the age of 40-60 years. Whereas in the variable body mass index was obtained the most in samples with a range of values of 18.5-25

\section{Table 1. Descriptive Analysis of The Patients}

\begin{tabular}{lcccc}
\hline \multicolumn{1}{c}{ Characteristics } & N & Mean \pm SD & Minimum & Maximum \\
\hline Age & 31 & $54.48 \pm 14.09$ & 21 & 82 \\
Body Mass Index & 31 & $24.79 \pm 3.09$ & 20 & 33.2 \\
Sacral Slope & 23 & $30.35 \pm 7.21$ & 19 & 42 \\
Pelvic Tilt & 23 & $32.91 \pm 5.51$ & 21 & 46 \\
Pelvic Incidence & 23 & $63.17 \pm 6.88$ & 55 & 84 \\
Intervention Level & 31 & $1.58 \pm 0.85$ & 1 & 4 \\
Pre-Operative Oswestry Score & 31 & $54.87 \pm 10.89$ & 32 & 80 \\
Post-Operative Oswestry Score & 31 & $13.32 \pm 5.49$ & 5 & 28 \\
Oswestry Score Difference & 31 & $42.06 \pm 11.52$ & 21 & 69 \\
Pre-Operative Rolland Morris Score & 31 & $13.90 \pm 2.89$ & 9 & 20 \\
Post-Operative Rolland Morris Score & 31 & $3.97 \pm 1.89$ & 2 & 9 \\
Rolland Morris Score Difference & 31 & $41.29 \pm 9.42$ & 25 & 60 \\
\hline
\end{tabular}

(ideal body weight). In the work variables found in the sample with the most work most of the activities sitting.

In this study operative variables were added in the form of fusion and the number of intervention levels. A total of 12 samples were interbody fused, and 19 samples were not fused, while the highest number of intervention levels were four levels.

After the sample underwent operative therapy, the sample returned to answer the Modified Oswestry disability scale questionnaire and the Rolland Morris disability scale. The questionnaire was filled out three days after the operation, when the sample had begun mobilization for the evaluation of disability and pain. The disability scale is added up and calculated the difference between before and after surgery.

\section{Patient Characteristics}

Descriptive analysis is intended to find a general description of research variables. This descriptive analysis is expected to provide a picture of the state of local government acceptance. To find a description for each variable, see the following Table 1.

\section{Statistical Analysis with the Modified Oswestry Disability Scale Regression Equations}

Regression equation is used to determine the form of the relationship between the independent variable with the dependent variable. By using SPSS for Windows 
ver 21.00 we get the regression model as shown in Table 3.

The regression equations obtained based on Table 3 are as follows:

$\mathrm{Y}=-0.208+0.410 \mathrm{X} 1+0.379 \mathrm{X} 2+$ $0.605 \mathrm{X} 3-0.763 \mathrm{X} 4+0.379 \mathrm{X} 5$

From the above equation can be interpreted as follows:

- Regression coefficient b1 of -0.208, meaning that the Modified Oswestry disability scale will decrease for each male gender. So the sample with the female gender has a difference in the scale of the Modified Oswestry disability that is greater than that of men.

- Regression coefficient b2 of 0.410, meaning that samples with an older age will experience a difference in the scale of the Modified Oswestry disability scale that is greater than the younger age.

- Regression coefficient b3 of 0.379, meaning that samples with sitting position work will experience a difference in the scale of the Modified Oswestry disability that is greater than other work positions.

- Regression coefficient b4 of 0.395, meaning that the difference in the scale of the Modified Oswestry disability scale will be greater if the sample has a high sacral slope.

- Regression coefficient b5 of -0.763, meaning that the difference in the scale of the Modified Oswestry disability scale will be even greater if the sample has a low pelvic incidence.

- Regression coefficient b6 of 0.379, meaning that the difference in the scale of the Modified Oswestry disability scale will be even greater if the sample is intervened with a large number of levels.

\section{Coefficient of Determination $\left(\mathbf{R}^{2}\right)$}

To determine the contribution of independent variables (gender, age, occupation, sacral slope, pelvic incidence,

Table 2. Descriptive Analysis of Patients Characteristics

\begin{tabular}{cccc}
\hline & Characteristics & f & $\%$ \\
\hline \multirow{2}{*}{ Job } & Sit down & 17 & 54.84 \\
& Stand up & 4 & 12.90 \\
Fusion & Weight Bearing & 10 & 32.26 \\
& No & 19 & 61.29 \\
Sex & Yes & 12 & 38.71 \\
& Female & 12 & 38.71 \\
Age & Male & 19 & 61.29 \\
& $<40$ yo & 4 & 12.90 \\
& $40-60$ yo & 15 & 48.39 \\
BMI & $>60$ to & 12 & 38.71 \\
& $18.5-25$ & 17 & 54.84 \\
& $25-30$ & 12 & 38.71 \\
& $>30$ & 2 & 6.45 \\
\hline
\end{tabular}

Table 3. Modified Oswestry Score Regression Results

\begin{tabular}{lccccc} 
& \multicolumn{2}{c}{ Standardized Coefficients } & \multirow{2}{*}{ df } & F & Sig. \\
\cline { 2 - 5 } & \multicolumn{1}{c}{ Beta } & Std. Error & & & \\
\hline Sex & -.208 & .095 & 1 & 4.736 & .041 \\
Age & .410 & .113 & 1 & 13.105 & .002 \\
Job & .379 & .110 & 2 & 11.998 & .000 \\
Sacral Slope & .605 & .287 & 1 & 4.427 & .048 \\
Pelvic Incidence & -.763 & .277 & 1 & 7.553 & .012 \\
Level of Intervention & .379 & .110 & 3 & 11.889 & .000 \\
\hline
\end{tabular}

and number of intervention levels) to the dependent variable (the Modified Oswestry disability scale) the adjusted $\mathrm{R} 2$ value is used, the use of Adjusted $\mathrm{R}^{2}$ is used because the independent variable is used more than 1 , so that if the addition of variable changes in determination is not too high.

Table 4. Coefficient of Determination

\begin{tabular}{ccc} 
R & R Square & $\begin{array}{c}\text { Adjusted R } \\
\text { Square }\end{array}$ \\
\hline 0.907 & 0.822 & 0.746 \\
\hline
\end{tabular}

From the analysis in Table 4, the adjusted $\mathrm{R}^{2}$ (coefficient of determination) result is 0.746 . This means that $74.6 \%$ of the Modified Oswestry disability scale variable will be influenced by the independent variables, namely gender, age, occupation, sacral slope, pelvic incidence, and the number of intervention levels. While the remaining $25.4 \%$ of the Modified Oswestry disability scale variable will be influenced by other variables not discussed in this study.

\section{Hypothesis Test}

Hypothesis testing is an important part of research, after data is collected and processed. Its main use is to answer the hypothesis made by the researcher.

Based on Table 3 the following results are obtained:

- F test between (Gender) with Y (Modified Oswestry disability scale) shows the value of $\mathrm{p}(0.041)<\alpha=0.05$ then the effect of sex on the Modified Oswestry disability scale is significant.

- $\quad$ F test between (Age) with Y (Modified Oswestry disability scale) shows the value of $p(0.002)<\alpha=0.05$ then the effect of age on the Modified Oswestry disability scale is significant.

- F test between (Occupation) and Y (Modified Oswestry disability scale) shows the value of $\mathrm{p}(0,000)<\alpha=$ 0.05 then the effect of work on the Modified Oswestry disability scale is significant.

- F test between (Sacral Slope) with Y (Modified Oswestry disability scale) shows the value of $\mathrm{p}(0.048)<\alpha=0.05$ then the effect of sacral slope on the Modified Oswestry disability scale is significant. 
- F test between (Pelvic Incidence) with Y (Modified Oswestry disability scale) shows the value of $p(0.012)<\alpha=0.05$ then the effect of pelvic incidence on the Modified Oswestry disability scale is significant.

- $\mathrm{F}$ test between (Number of Intervention Levels) and Y (Modified Oswestry disability scale) shows the value of $p(0,000)<\alpha=0.05$ then the effect of the number of intervention levels on the Modified Oswestry disability scale is significant

\section{Regression Analysis of Rolland Morris Score Reduction Categories Regression Equation}

Regression equation is used to determine the form of the relationship between the independent variable with the dependent variable. By using SPSS for Windows ver 21.00 we get the regression model as in Table 5.

The regression equations obtained based on Table 5.5 are as follows:

$\mathrm{Y}=0,300 \mathrm{X} 1+0,381 \mathrm{X} 2+0,312 \mathrm{X} 3$

From the above equation can be interpreted as follows:

- Regression coefficient b1 of 0.300, meaning that samples with older age will experience a difference in the Rolland Morris disability scale that is greater than with younger ages.

- Regression coefficient b2 of 0.381, meaning that samples with sitting position work will experience a difference in Rolland Morris disability scale that is greater than other work positions.

- Regression coefficient b3 of 0.312, meaning that the difference in the Rolland Morris disability scale will be even greater if the sample has a higher number of intervention levels.

Table 5. Rolland Morris Score Regression Results

\begin{tabular}{ccccccc} 
& \multicolumn{2}{c}{ Standardized Coefficients } & df & F & Sig. \\
\cline { 2 - 3 } & Beta & Std. Error & & & \\
\hline Age & .300 & .143 & 1 & 4.402 & .046 \\
Job & .381 & .141 & 1 & 7.289 & .012 \\
Level of Intervention & .312 & .146 & & 4 & 4.539 & .020 \\
\hline
\end{tabular}

Dependent Variable: ROLLAND MORRIS

\section{Coefficient of Determination $\left(\mathbf{R}^{2}\right)$}

To determine the contribution of the independent variable (age, occupation and number of intervention levels) to the dependent variable (Rolland Morris disability scale) the adjusted $R^{2}$ value is used, the use of adjusted $\mathrm{R}^{2}$ is because the independent variable is used more than 1 , so that if there is an additional variable the determination changes is not too high.

Table 6. Coefficient of Determination

\begin{tabular}{ccc}
\hline R & R Square & Adjusted R Square \\
\hline 0.760 & 0.578 & 0.513 \\
\hline
\end{tabular}

From the analysis in Table 6 we get the adjusted $\mathrm{R}^{2}$ (coefficient of determination) result of 0.513 . This means that $51.3 \%$ of the Rolland Morris disability scale variable will be influenced by the independent variables, namely age, occupation, and the number of intervention levels. While the remaining $48.7 \%$ of the Rolland Morris disability scale variable will be influenced by other variables not discussed in this study.

\section{Hypothesis Testing}

Hypothesis testing is an important part of research, after data is collected and processed. Its main use is to answer the hypothesis made by the researcher.

Based on Table 5 the following results are obtained:

- F test between (age) with Y (Rolland Morris disability scale) shows the value of $\mathrm{p}(0.046)<\alpha=0.05$ then the effect of age on the Rolland Morris disability scale is significant.

- F test between (work) with Y (Rolland Morris disability scale) shows the value of $p(0.012)<\alpha=0.05$ then the effect of work on the Rolland Morris disability scale is significant.
- F test between (number of intervention levels) and Y (Rolland Morris disability scale) shows the value of $p(0.020)<\alpha=0.05$, the effect of the number of intervention levels on the Rolland Morris disability scale is significant.

\section{DISCUSSION}

\section{Anthropometric factor}

From the sex factor in this study, the majority of the samples were 19 male samples, and 12 female samples. However, the results of the statistical analysis of the regression on the Modified Oswestry disability scale found that women had a disability scale difference that was greater than men, with a $p$ value $(0.041)$ indicating that gender had a strong influence on the Modified Oswestry disability scale. This indicates that in women, the disability and pain scale was significantly reduced after surgery.

Women experiences more chronic musculoskeletal pain, compared to men. And this significant difference might be due to the fact that women are better able to withstand pain and choose not to report symptoms. ${ }^{7}$ Other studies have shown that women with low back pain who have high cortisol levels show low lumbar strength. Higher levels of this catabolic hormone in the bloodstream may be associated with higher levels of deterioration and dehydration which affect the intervertebral discs especially in the elderly and high cortisol in women is also associated with pain intensity presented by patients. ${ }^{8}$

Women are said to have a greater risk for LBP, which is $(54.91 \%)$ greater than men (45.09\%). However, many studies have stated that the prevalence is more common in men in various developing countries where the majority of men are manual laborers and pain related to work activities. ${ }^{9}$ This suggests that degenerative lumbar disease may have a higher prevalence in men, but a higher pain intensity was observed in a female sample.

In this study also obtained the majority of samples obtained in the age range 40-60 years. This is consistent with most other studies which state that the majority of the population of patients with low back pain is above the fourth decade. However, there is a shift in the average age of 21.54 
in the female sample. From the results of the regression statistical analysis it is found that the influence of older age will provide a large scale disability difference in Modified Oswestry and Rolland Morris. The age factor also had a significant influence on the Modified Oswestry scale with $\mathrm{p}$ (0.002) and Rolland Morris with $\mathrm{p}$ (0.046).

In a study conducted by Su et al. among 357 patients the incidence of LBP was most common in the age group between 40-59 years and around (48.17\%) followed by groups $60-79,20-39$ and $\geq 80$ years with $21.29 \%, 17.65 \%$ and $12.89 \%$ respectively. ${ }^{10}$

The shift in the average age of patients, especially in the sample of women becoming younger is likely due to some of them being a work activity factor that makes humans less active in their daily lives, and most of them are pregnancy factors. Pregnancy can predispose to persistent low back pain. In one study it was stated that $50-60 \%$ of pregnant women experience low back pain with acute onset $^{11}$ and $1 / 3$ of patients will experience work, sleep, and daily activities. ${ }^{12}$ Lower back pain in pregnant women often persists after giving birth and a study says that lower back pain during pregnancy will be a strong risk factor for persistent low back pain.?

In this study, the majority of patients had an ideal body mass index (18.525) of 17 of 31 samples, and body mass index did not have a correlation to the difference in scores on Modified Oswestry or Rolland Morris. This study is in line with the results of research from Winn and Youmans which states that an increase in body mass index is strongly associated with an increase in the prevalence of low back pain, but not with frequency or severity. ${ }^{13}$ While a study from LeboeufYde which states that body mass index is a weak risk factor for the incidence of low back pain. The results of research on the effect of body mass index on low back pain in various countries showed varied results. There are studies that say that obesity or a high body mass index is associated with an increased incidence of low back pain. ${ }^{14}$

The most important mechanism of low back pain is due to spinal overload, but the exact mechanism is still unknown. For example, patients undergoing surgery for lumbar disc herniation are higher and more severe for lower back pain than patients from the general population when correlated with age and sex. Likewise, patients discharged from hospitals with a diagnosis of disc hernias are also more likely to have a higher body mass index, and a high body mass index has been correlated with the prevalence, extent, and severity of lumbar intervertebral disc disease. Other mechanisms found, including psychological effects, decreased physical activity, systemic inflammation due to the release of adipocytokines, and atherosclerosis result in decreased blood flow and nutrition to the disc. ${ }^{13}$

In this study the body mass index factor does not have a significant effect on the disability scale of Modified Oswestry and Rolland Morris. This is because most of the sample has an ideal body weight. Body mass index in some countries is an influential prevalence factor, but it is limited to the category of overweight or obesity.

In this study the work factor is divided into three categories based on the most activities carried out and obtained three categories, namely sitting, standing, and lifting weights. the majority of samples have jobs that require sitting, ie 17 of 31 samples. And 10 samples from this study with work that required to lift weights. From the results of statistical analysis with regression, it was found that in patients with the majority of sitting activities, the disability scale was reduced by the Modified Oswestry and Rolland Morris disability scales. And have a significant influence with $\mathrm{p}(0.00)$ on the Modified Oswestry disability scale and $\mathrm{p}(0.012)$ on Rolland Morris.

In discussing the management and causes of low back pain, the role of physical activity is an important research problem in controlling the risk of low back pain. Studies focusing on the relationship between back pain and physical activity (such as work, recreation, and sportsrelated activities) have produced results that are compatible with U-shaped models, addressing the adverse effects of sedentary behaviour and heavy levels of physical activity. ${ }^{15}$

Biomechanical burden seems to be the most important occupational factor that predicts recurrent low back pain and sick leave associated with back disorders. Exposure to activities that use a lot of hands (manual work) is considered an important risk factor, consistently associated with back disorders related to work. People in these jobs are significantly more likely to get sick leave because of back pain. Several studies have assessed aspects of physical burden as a possible risk factor for LBP. A common problem in this study is the accurate assessment of physical activity in relation to its intensity and frequency. ${ }^{16}$

In 1999, Hoogendoorn et al. systematically review aspects of physical load during work and leisure as risk factors for back pain. ${ }^{17}$ Heavy physical work, manual handling, and a body that bends a lot and protects are found to be risk factors for back pain. No evidence was found for specific activities such as standing or walking, sitting, exercising, and physical activity in total free time. However, exposure to physical loads is not limited to one specific back threatening activity, but includes compilation of activities, such as flexion, rotation, lifting, transporting, and withdrawal. The results of a crosssectional investigation of the prevalence of risk factors for chronic diseases in a randomly selected sample from the Dutch population indicate that a reduction in physical burden could theoretically reduce the burden of lower back pain by $13-18 \%{ }^{16}$

In one systematic review study in the Netherlands, there is strong evidence that heavy physical workloads and exposure to non-ergonomic positions are risk factors for back pain. Moderate to strong risk estimates exist for manual work, which is defined as the sum of 'lifting and carrying loads' and 'pushing or pulling', bending and twisting. These results are consistent with the results in a review by Hoogendoorn et al. ${ }^{17}$ Inconsistent findings were found for physical activity variables in leisure, exercise and physical exercise. With respect to the relationship between physical burden and low back pain, research is mainly focused on physical activity at work. Large studies that focus on daily physical activity habits in relation to LBP are lacking. But this study emphasizes the importance of considering the complex etiology and multi-dimensional structure of risk factors. It can be assumed 
that there is no bivariate relationship, but multivariate, so physical activity is one but not the only factor. ${ }^{16}$

Another systematic review carried out in India concluded that risk factors met the criteria but were inconsistent. 'Standing / walking $>2$ hours' and 'lifting / moving > 25 lbs' were considered very significant because they were identified by longitudinal studies in participants with 'no history of low back pain'. However, they are not consistently reported by research. ${ }^{15}$

Prolonged sitting position has strong implications for the musculoskeletal system, the increase in musculoskeletal pain is reported to increase with sitting time. In the sitting position there is an increase in intradiscal pressure from the flexi position in the spine. The sitting position compression force on L4-L5 experienced can be higher than the average $500 \mathrm{~N}$ compared to standing which results in shear forces on the anterior-posterior aspect. $^{18}$

In this study shows that sedentary lifestyle and occupational risk factors with the majority of sitting activities become a strong influence factor compared to the sitting position and lifting weights.

\section{Anatomical Factors}

The anatomic factor assessed in this study is the pelvic parameter which has an influence on the degree of low back pain. Pelvic parameters include:

1. Pelvic incidence: The angle measured from the line perpendicular to the endplate $S 1$ and the line drawn to the centre of the femoral head. Normally around 50 degrees.

2. Pelvic tilt: The angle measured from the line drawn from the endplate $\mathrm{S} 1$ to the centre of the femoral head and the line that is perpendicular to the horizontal film.

3. Sacral slope: The angle measured from a line parallel to endplate $S 1$ and a horizontal line

The relationship between pelvic incidence, sacral slope, and pelvic tilt is defined by the following equation: pelvic incidence $=$ sacral slope + pelvic tilt

In this study, the value of sacral slope with a mean value of $30.35 \pm 7.21$, pelvic tilt with a mean value of $32.91 \pm 5.51$, and pelvic incidence with a mean value of $63.17 \pm 6.88$.
From the regression results it is found that the high sacral slope has a high difference in value on the Modified Oswestry disability scale but does not have an influence on the Rolland Morris disability scale. Sacral slope has a significant influence on the decrease in the Modified Oswestry disability scale with $\mathrm{p}$ (0.048)

The regression results also show that a low pelvic incidence has a high difference in value on the Modified Oswestry disability scale but does not have an influence on the Rolland Morris disability scale. Pelvic incidence has a significant effect on the decrease in the scale of disability Modified Oswestry with $\mathrm{p}$ (0.012)

Pelvic incidence remains the same after skeletal maturity is reached. Individuals with a positive sagittal imbalance in their pelvic retrovert in an effort to maintain sagittal alignment, flatten the sacral slope and increase pelvic tilt. Understanding these parameters and their relationship is very important for planning surgical correction of abnormalities. The ideal lumbar lordosis must be within 9 degrees of pelvic incidence; thus, patients with greater pelvic incidence may require greater surgical correction for ideal alignment. Measuring sacral slope and pelvic tilt allows the surgeon to estimate the amount of pelvic compensation used by the patient and provides a more complete picture of the severity of the spine than just assessing the sagittal vertical axis alone. ${ }^{13}$

Normal spinal alignment is characterized by cervical lordosis, thoracic kyphosis, and lumbar lordosis, which together produce sagittal balance, in which the body's centre of gravity is maintained in the axis above the pelvis and legs. Because of this balance, minimal muscle power is needed to defy gravity in an upright posture. In adults, normal sagittal angulation is generally defined as 30 to 40 degrees of cervical lordosis, 20 to 40 degrees of thoracic kyphosis, and 20 to 45 degrees of lumbar lordosis. ${ }^{13}$ In a study conducted by Krol, et al., Showed that shortening / contraction of the hip flexor muscles causes anterior pelvic tilt, but this relationship was not statistically significant, regardless of age, both in people with and without pain. Krol also confirmed the relationship between lumbar lordosis and pelvic tilt where in patients with increased lordosis, an increase in pelvic tilt values was found (Król, Polak, Szczygieł et al., 2017). The study also suggests something that is in line with our study in which the mean value of the degree of pelvic tilt is directly proportional to lower back pain. In a meta-analysis study conducted by Laird, et al., It was found that the anterior pelvic tilt had a greater role in the posterior inclination. This can be considered for muscle stretching as a routine procedure during LBP patient care and posture correction to improve the degree of tilt. The movement of the patient during the nurse should be considered to reduce pain due to incorrect posture based on the tilt of the pelvis. ${ }^{19}$

Another study was carried out by Chaleat-Valayer, et al., Extensively on 198 cases of low back pain and 709 normal patients. In this cohort study, it was found that sacral slope and pelvic incidence had a significant effect where the value of pelvic incidence in lower back pain was smaller by two degrees compared to the control group. The interesting thing about this research in relation to our study is the role of sacral slope as a factor that is more closely related to lower back pain where the value of sacral slope in the pain group is 2.9 degrees smaller than the control but gives a greater significance value. ${ }^{20}$

The results of this study are in line with the results we found where the value of sacral slope and pelvic incidence exerts influence on the incidence of low back pain. Correction of pelvic parameters should be taken into account in the management of low back pain given the significant results in the small degree of difference. This small difference value should be considered a bias in lower back pain studies where the value of the data obtained has a strong potential for patient selection.

\section{Intervention Factors}

The main goal of surgical intervention is to decompress the nerve structure that is being compressed. Theoretically, surgery will eliminate symptoms and improve function. Specific details of the operative action approach vary according to location, number of segments affected, spinal deformity or instability, history of surgery, and surgeon's preference. Various approaches to achieving decompression 
include traditional laminectomy, bilateral laminotomy, bilateral decompression through unilaterallaminotomy, and various forms of laminoplasty. ${ }^{21}$ Decompression measures generally focus on the goal of relieving foot symptoms (claudication or radiculopathy) and less on increasing the accompanying back pain. Therefore, although back pain improves, the increase in leg pain is usually more significant. Patients with dominant symptoms of leg pain have better surgical results and a greater relative increase in outcome than non-operative approaches. This group of patients also had better results compared to patients with back pain as a dominant symptom or in an equal degree. ${ }^{21}$

Indications for lumbar spinal fusion are spondylolisthesis, unstable spinal stenosis syndrome (degenerative spondylolisthesis, degenerative scoliosis), and in patients with segmental instability. ${ }^{22}$ Other studies show that indications for fusion include iatrogenic instability, isthmic spondylolisthesis, kyphosis, stenosis in previously decompressed segments, stenosis adjacent to lumbar segments previously fused, dynamic instability radiographically proven with pain or neurological findings, scoliosis adult, and mechanical low back pain. Relative indications for the use of spinal instrumentation include correction of deformity, recurrent spinal stenosis with instability, degenerative spondylolisthesis, adjacent segment stenosis with instability, and multiple-level fusion. Fusion is rarely indicated in routine discectomy, abnormal radiological findings without specific findings (such as degenerative disc disease), facet joint syndrome, failed back surgery, or stable spinal stenosis. ${ }^{23}$

The act of decompression or decompression surgery with fusion is a controversy that has long been discussed. Many types of lumbar fusion techniques go through different approaches including posterior / posterolateral lumbar fusion, posterior lumbar interbody fusion, transforaminal lumbar interbody fusion (TLIF) and oblique lumbar interbody fusion (OLIF). At present statistically the number of decompression measures plus fusion for lumbar stenosis continues to increase while decompression alone decreases. ${ }^{24}$ One of the main controversies about surgery for the spine is the role of spinal fusion. Spinal arthrodesis with the aim of achieving spinal fusion is generally recommended for spinal stenosis associated with degenerative spondylolisthesis, recurrent stenosis after decompression, instability, or previous scoliosis. Recent clinical practice guidelines recommend that if there is no deformity or instability, lumbar fusion has not been shown to improve outcomes in patients with isolated stenosis, and therefore is not recommended. ${ }^{25}$ Likewise, guidelines from the North American Spine Society recommend that in the absence of scoliosis or spondylolisthesis, only decompression surgery is recommended for patients with dominant foot symptoms without instability (grade B). ${ }^{25,26}$

Because there is a lack of evidence for the benefits of fusion, this technique should be limited to lower back pain patients who have spinal instability, spinal deformity, or spinal damage caused by trauma, tumour and infection, or spinal foramen stenosis with compressed nerves caused by the collapse of the disc after the surgery. ${ }^{27}$

The reason for surgery is chosen to regulate intraspinal pressure, blood flow, and metabolic status of nerve structures by decompressing nerve elements and reducing the inflammation process. ${ }^{28}$ Options for conservative management in LSS which include medication, physiotherapy, spinal injection, lifestyle modification, and multidisciplinary rehabilitation can only benefit in the early development of the disease with mild symptom severity. ${ }^{29}$ Symptoms of a patient that worsen can increase pain to moderate and severe pain. Surgery is performed to prevent further deterioration in this case. It is important to emphasize that motor weakness, sphincter dysfunction, or other signs such as acute cauda equina syndrome, are highly recommended for evaluation of surgical procedures with the choice of a decompression surgery approach as an appropriate first step. Early studies show the surgical approach is superior to conservative treatment. This is a consideration for patients to undergo further surgery if unsatisfactory results are achieved in the initial conservative treatment. This finding contradicts recent research by the COCHRANE database which systematically reviews surgical versus non-surgical treatments for. From this research it can be seen that there are still no updated recommendations and there is no superiority between management options that require the surgeon to be careful in informing patients about some of the available treatment options. ${ }^{30}$ In a study conducted by Shen, et al., On 438 patients, no significant difference was seen between decompression surgery without and with fusion based on the Oswestry Disability Index (ODI) and postoperative observations within two years. The level of patient satisfaction also has similar results between groups. One thing to note in fusion surgery is the increased duration of surgery, intraoperative bleeding, and length of stay in the hospital. ${ }^{31}$ This should be considered in the selection of operative measures in low back pain where fusion can be said to have greater potential in increasing morbidity. More attention should be given to vulnerable population groups such as the elderly or other patients with comorbidities that might make it difficult to postoperative care.

The choice of surgery with fusion at this time remains the choice in patients with low back pain despite the controversy from the above discussion. Research by Phillips, et al., Systematically has shown that these actions are operative management options to significantly reduce pain and improve daily function in patients with low back pain compared to non-operative measures. One other consideration worthy of consideration in the selection of operative measures is the duration of the results of pain and function improvement in lower back pain patients who are operated on can last longer than other options. One interesting thing in this research is the absence of differences resulting from the selection of fusion techniques. ${ }^{32}$ Further research needs to be done on the benefits of the choice of fusion surgery techniques that can be done.

In this study, the act of fusion had no influence on the outcome of operating outcomes which were evaluated using either the Modified Oswestry or Rolland Morris disability scale. The number of intervention levels has a significant effect on operating outcomes. Evaluations using the Modified Oswestry and Rolland Morris disability scales indicate that the greater number of interventions carried 
out will increase the difference on both disability scales. This can imply that the degree of pain decreases significantly with the number of vertebral levels intervened. This is likely due to the effect of decompression carried out in line with the level of intervention.

\section{CONCLUSIONS}

There is an effect of sex, age, sitting working position and sacral slope factors on the outcome of surgical procedures in the degenerative lumbar patient evaluated with the Modified Oswestry disability scale. Whereas the age and sitting work position have an influence in surgical outcome in the patients evaluated with Rolland Morris scale. Number of intervention levels tend to have a significant effect on disability score difference.

\section{ACKNOWLEDGMENTS}

Non declared.

\section{DECLARATIONS}

\section{FUNDING}

No specific funding.

\section{CONFLICT OF INTEREST}

Authors have no conflict of interest.

\section{ETHICAL APPROVAL}

\section{AUTHORS' CONTRIBUTIONS}

Authors took part in design of the study and contributed to data collection. YFP did literature review and drafted the manuscript. $\mathrm{AHB}$ and $\mathrm{HB}$ made critical revisions to the manuscript and all agree to accept equal responsibility for accuracy of the contents of this article.

\section{REFERENCES}

1. Allegri, M. et al. Mechanisms of low back pain: A guide for diagnosis and therapy [version 1; referees: 3 approved]. F1000Research 5, 1-11 (2016).

2. Aalto, T. J. et al. Preoperative predictors for postoperative clinical outcome in lumbar spinal stenosis: Systematic review. Spine (Phila. Pa. 1976). 31, 648-663 (2006).

3. Samartzis, D. et al. A population-based study of juvenile disc degeneration and its association with overweight and obesity, low back pain, and diminished functional status. J. Bone Jt. Surg. - Ser. A 93, 662-670 (2011).

4. Atlas, S. J., Keller, R. B., Chang, Y., Deyo, R. A. \& Singer, D. E. Surgical and Nonsurgical Management of Sciatica Secondary to a Lumbar Disc Herniation. Spine (Phila. Pa. 1976). 26, 1179-1187 (2001).

5. Delitto, A. et al. Low Back Pain Clinical Practice Guidelines Linked to the International Classification of Functioning, Disability, and Health from the Orthopaedic Section of the American Physical Therapy Association. J. Orthop. Sports Phys. Ther. 42, (2012).

6. Fritz, J. M. \& Irrgang, J. J. A comparison of a modified Oswestry Low Back Pain Disability Questionnaire and the Quebec Back Pain Disability Scale. Phys. Ther. 81, 776-788 (2001).

7. Battié, M. C., Videman, T. \& Parent, E. Lumbar disc degeneration: Epidemiology and genetic influences. Spine (Phila. Pa. 1976). 29, 2679-2690 (2004).

8. Eichler, J., Rachinger-Adam, B., Kraft, E. \& Azad, S. C. Efficacy of biofeedback in patients with chronic low back pain: Impact on pain intensity, psychological factors and stress markers. Schmerz 33, 539-548 (2019).

9. Heuch, I., Hagen, Kurt, Heuch, I., Nygaard, $\varnothing$. \& Zwart, J. The Impact of Body Mass Index on the Prevalence of. Spine (Phila. Pa. 1976). 35, 764-768 (2010).

10. Su, C. A., Kusin, D. J., Li, S. Q., Ahn, U. M. \& Ahn, N. U. The Association between Body Mass Index and the Prevalence, Severity, and Frequency of Low Back Pain. Spine (Phila. Pa. 1976). 43, 848-852 (2018).

11. Ostgaard, H. C., Andersson, G. B. J. \& Karlsson, K. Prevalence of Back Pain in Pregnancy. (1991).

12. Kristiansson, P., Svärdsudd, K. \& Von Schoultz, B. Back pain during pregnancy: A prospective study. Spine 21, 702-709 (1996).

13. Winn, H. Youmans \& Winn Neurological Surgery. (Elsevier, 2017).

14. Leboeuf-Yde, C., Kyvik, K. O. \& Bruun, N. H. Low back pain and lifestyle. Part II--Obesity. Information from a population-based sample of 29,424 twin subjects. Spine 24, 779-83; discussion 783-4 (1999).

15. Tissot, F., Messing, K. \& Stock, S. Studying the relationship between low back pain and working postures among those who stand and those who sit most of the working day. Ergonomics 52, 1402-1418 (2009).

16. Heneweer, H., Staes, F., Aufdemkampe, G., Van Rijn, M. \& Vanhees, L. Physical activity and low back pain: A systematic review of recent literature. Eur. Spine J. 20, 826-845 (2011).

17. Hoogendoorn, W. E. et al. High physical work load and low job satisfaction increase the risk of sickness absence due to low back pain: Results of a prospective cohort study. Occup. Environ. Med. 59, 323-328 (2002).

18. Aggarwal, N., Anand, T., Kishore, J. \& Ingle, G. K. Low back pain and associated risk factors among undergraduate students of a medical college in Delhi. Educ. Heal. Chang. Learn. Pract. 26, 103-108 (2013).

19. Król, A., Polak, M., Szczygieł, E., Wójcik, P. \& Gleb, K. Relationship between mechanical factors and pelvic tilt in adults with and without low back pain. J. Back Musculoskelet. Rehabil. 30, 699-705 (2017).

20. Chaléat-Valayer, E. et al. Sagittal spino-pelvic alignment in chronic low back pain. Eur. Spine J. 20 Suppl 5, 634-640 (2011).

21. Lurie, J. \& Tomkins-Lane, C. Management of lumbar spinal stenosis. BMJ 352, (2016).

22. Hanley, E. N. The indications for lumbar spinal fusion with and without instrumentation. Spine 20, 143-153 (1995).

23. Detwiler, P. W., Marciano, F. F., Porter, R. W. \& Sonntag, V. K. H. Lumbar stenosis: indications for fusion with and without instrumentation. Neurosurg. Focus 3, E6 (2008).

24. Mobbs, R. J., Phan, K., Malham, G., Seex, K. \& Rao, P. J. Lumbar interbody fusion: techniques, indications and comparison of interbody fusion options including PLIF, TLIF, MI-TLIF, OLIF/ATP, LLIF and ALIF. J. spine Surg. (Hong Kong) 1, 2-18 (2015).

25. Rittenberg, J. D. \& Ross, A. E. Functional rehabilitation for degenerative lumbar spinal stenosis. Phys. Med. Rehabil. Clin. N. Am. 14, 111-120 (2003).

26. Peul, W. C. \& Moojen, W. A. Fusion for lumbar spinal stenosis - safeguard or superfluous surgical implant? N. Engl. J. Med. 374, 14781479 (2016).

27. Wu, A.-M., Tong, T.-J. \& Wang, X.-Y. A rethink of fusion surgery for lumbar spinal stenosis. J. Evid. Based. Med. 9, 166-169 (2016).

28. Ainslie, P. N. \& Brassard, P. Why is the neural control of cerebral autoregulation so controversial. F1000Prime Rep. 6, 5-10 (2014).

29. Ammendolia, C. et al. Nonoperative treatment of lumbar spinal stenosis with neurogenic claudication: A systematic review. Spine (Phila. Pa. 1976). 37, 609-616 (2012).

30. Zaina, F., Tomkins-Lane, C., Carragee, E. \& Negrini, S. Surgical versus non-surgical treatment for lumbar spinal stenosis (Cochrane review) [with consumer summary]. Cochrane Database Syst. Rev. 2016;Issue 1 (2016). doi:10.1002/14651858. CD010264.pub2.www.cochranelibrary.com

31. Shen, J., Xu, S., Xu, S., Ye, S. \& Hao, J. Fusion or not for degenerative lumbar spinal stenosis: A meta-analysis and systematic review. Pain Physician 21, 1-7 (2018).

32. Phillips, F. M., Slosar, P. J., Youssef, J. A., Andersson, G. \& Papatheofanis, F. Lumbar spine fusion for chronic low back pain due to degenerative disc disease: A systematic review. Spine (Phila. Pa. 1976). 38, 409-422 (2013).

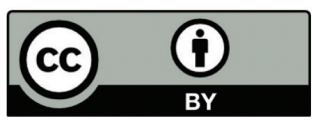

This work is licensed under a Creative Commons Attribution 